\title{
Characterization of Biocompatible Poly(Ethylene Glycol)-Dimethacrylate Hydrogels for Tissue Engineering
}

João Lopes ${ }^{1, a}$, Rita Fonseca ${ }^{2, b^{*}}$, Tânia Viana ${ }^{2, c}$, Cristiana Fernandes ${ }^{2, d}$, Pedro Morouço ${ }^{2, \mathrm{c}}$, Carla Moura ${ }^{2, \mathrm{~d}}$ and Sara Biscaia ${ }^{2, e}$

${ }^{1}$ Escola Superior de Tecnologia e Gestão, Polytechnic Institute of Leiria, Portugal, ${ }^{2}$ Centre for Rapid and Sustainable Product Development, Polytechnic Institute of Leiria, Portugal

a2140464@my.ipleiria.pt, bana.r.fonseca@ipleiria.pt, ctania.viana@ipleiria.pt, dcristiana.fernandes@ipleiria.pt, epedro.morouço@ipleiria.pt, ${ }^{\mathrm{f}} \mathrm{c}$ carla.moura@ipleiria.pt, gsara.biscaia@ipleiria.pt

${ }^{*}$ corresponding author

Keywords: hydrogel; photoinitiator; poly(ethylene glycol)-dimethacrylate, mechanical properties, FTIR.

\begin{abstract}
Tissue Engineering depends on broadly techniques to regenerate tissues and/or organ functions. To do so, tailored polymeric and/or hydrogel scaffolds may be used to ensure the appropriate regeneration. Hydrogels are suitable materials for constructing cell-laden matrices as they can be produced with incorporation of cells and rapidly cross-linked in situ through photopolymerisation reactions. Measurement of the polymerization degree, as well as resistance to compression and water retention are fundamental tests to evaluate the characteristics of hydrogels. In this work, free-radical polymerisation of poly(ethylene glycol)-dimethacrylate (PEGDMA) in UV light was assessed. Several hydrogels with different photoinitiator and water contents were produced to evaluate their influence on hydrogels behaviour. Experiments showed that variations on water and photoinitiator content induce changes in the physical and chemical behaviour of hydrogels. As it was found, water content prevents polymerisation to occur and reduces the mechanical properties of hydrogels weakening them. Furthermore, differences were found in varying water content from 15 to $30 \%$, since this increase turned hydrogels more fragile and increase their stabilization time for water retention.
\end{abstract}

\section{Introduction}

Strategies in Tissue Engineering (TE) and Regenerative Medicine (RM) seek to restore and maintain normal function of injured or diseased tissues. These are interdisciplinary areas, with several specificities and requirements related to cell biology, materials science and engineering, with the purpose of developing substitutes that mimic anatomical and functional features of native tissues [1]. New synthetic biomaterials are being developed at rapid pace for use as threedimensional (3D) extracellular microenvironments. The aim is to mimic the regulatory characteristics of natural extracellular matrices (ECMs) and ECM-bound growth factors [2]. All the specifications for the biomaterials used in these processes are critical, and such success is achievable with a combination of optimal mechanical properties and maximal chemical and biological inertness, simulating as closely as possible the biochemical, biomechanical and morphological characteristics that cells normally experience [3]. It is mandatory that the biomaterial has appropriate elasticity, biocompatibility and an optimal architecture replicating cell niche, capable of constantly changing the microenvironment. Depending on the prevailing circumstances, the material may require features of self-assembly and injectability, an appropriate degradation profile, and should be compatible with imaging processes/modalities [3, 4].

The cell microenvironment has been focused on hydrogels-based tissues, as ECM-based structures, biopolymers and peptide hydrogels. Particular attention is being given to macro- and nanoscale architecture and also self-assembly [3]. A TE matrix can provide an environment marked by its 
biomechanical structure, its structural and adhesion molecules and, its diffusible biomolecular character, such as growth factors and chemokines [2]. This template, which comprises the biomaterial, the architecture and the biomolecules, has to simulate the cell niche and may be preformed or injectable [5].

Hydrogels have been used in many applications for injectable approaches in cell therapy and TE, which allow the repair of tissue defects or injection of cells for achieving cellular function [6]. A hydrogel is a 3D network formed by cross-linking water soluble polymers to form an insoluble hydrophilic polymer network, swollen by water, its major component [1]. In the past decade, progress in creating 3D cellular microenvironments using hydrogels has obtained similar elasticity to that of native tissues. The structure and composition of hydrogels can be tailored to bear the suitable chemical, biological, and physical cues that encourage the development of tissue constructs [7]. Poly(ethylene glycol) dimethacrylate (PEGDMA) has been widely investigated as a hydrogel in the field of coatings, adhesives, dental and cartilage repair [8], and also for encapsulating, delivering and seeding of cells [9-13] and bioactive molecules [14, 15], and even as a bioink suitable for bioprinting purposes, synthesis of new tissue [16] and as a biomaterial for bone regeneration [17, 18].

This work focused on the development of PEGDMA membranes, and its characterization through the assessment of free-radical photopolymerisation, water retention and mechanical testing. Additionally, it was examined the influence of water and photoinitiator content, to analyse hydrogels behaviour.

\section{Materials and Methods}

Materials. To evaluate the water and photoinitiator influence on hydrogel membranes behaviour two groups of samples were developed. Hydrogel samples with different concentrations of photoinitiator $(0.5,1$ and $1.5 \mathrm{wt} \%)$ and distilled water $(0,15$ and $30 \mathrm{wt} \%)$ were synthetized and organized according to their composition as shown in Table 1. Each membrane is composed of a photopolymerised solution made of PEGDMA ( $M w$ 750) and 2-hydroxy-4'-(2-hydroxyethoxy)-2methylpropiophenone (Irgacure 2959), both supplied by Sigma-Aldrich.

Table 1- Constituents of prepolymerised hydrogel membranes. Different photoinitiator Irgacure 2959 concentrations (wt/wt) were prepared in a mixture solution of PEGDMA 750 with water content in different proportions.

\begin{tabular}{cccc}
\hline Hydrogel & PEGDMA 750 (\%) & Water $(\%)$ & Irgacure 2959 (\%) \\
\hline $\mathrm{A}_{1}$ & 100 & 0 & 0.5 \\
$\mathrm{~A}_{2}$ & 85 & 15 & \\
$\mathrm{~A}_{3}$ & 70 & 30 & 1.0 \\
\hline $\mathrm{B}_{1}$ & 100 & 0 & \\
$\mathrm{~B}_{2}$ & 85 & 15 & 1.5 \\
$\mathrm{~B}_{3}$ & 70 & 30 & \\
\hline $\mathrm{C}_{1}$ & 100 & 0 & \\
$\mathrm{C}_{2}$ & 85 & 15 & \\
$\mathrm{C}_{3}$ & 70 & 30 & \\
\hline
\end{tabular}

Synthesis of Hydrogel membranes. Hydrogel membranes were obtained by a free-radical photopolymerisation reaction between PEGDMA and photoinitiator Irgacure 2959, sensible to ultraviolet radiation (UV). A total of 9 mixtures, according to table 1, were prepared by adding photoinitiator to the previously PEGDMA in a desired amount of distilled water. The batches were placed onto a magnetic stirrer (400 rpm), for 1 hour, until a homogeneous mixture was achieved. All this process was made reducing the exposure to UV light at the most. Samples were left overnight, and then transferred into Petri dishes $(\mathrm{n}=9)$ until obtain a mass of $\sim 23 \mathrm{~g}$ and a $4 \mathrm{~mm}$ thickness. Each Petri dish was submitted to UV radiation with an irradiance of $\sim 9 \mathrm{~mW} / \mathrm{cm}^{2}$ for $15 \mathrm{~min}$. 
For each sample (A1 to C3), small cylinders with a diameter of $14 \mathrm{~mm}$ were removed from photopolymerised PEGDMA mixtures and stored for FTIR-ATR, swelling studies and compression resistance tests. In Fig. 1a is possible to observe one cylinder sample and in Fig. 1b a scheme showing 9 different cylinders from different obtained samples.
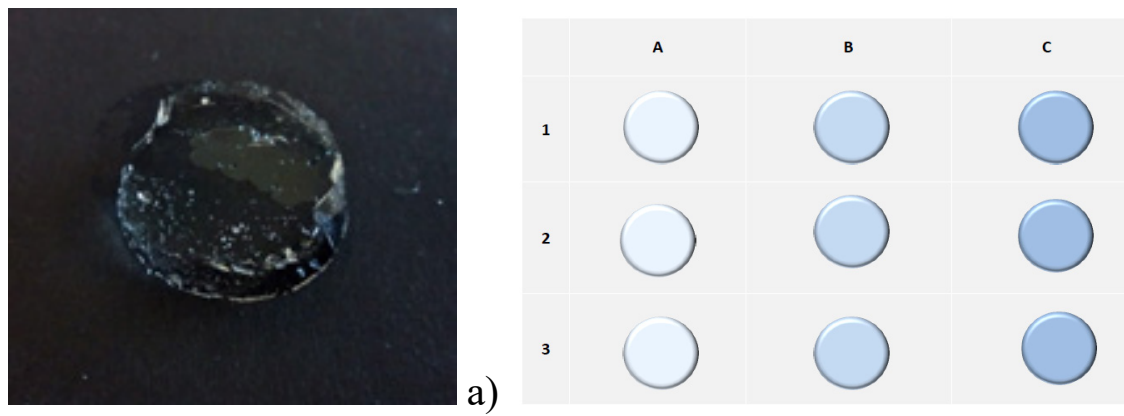

b)

Fig. 1- a) Hydrogel cylinder removed from a photopolymerised sample. b) Worksheet describing the organization of photopolymerised samples according to their content. Vertical alignment: A, B, C (photoinitiator content (wt\%): 0.5, 1.0, 1.5 respectively). Horizontal alignment: 1, 2, 3 (water content (wt\%): $0 ; 15 ; 30$ respectively.

\section{Characterisation Methods}

- Fourier Transform Infrared with Attenuated Total Reflectance Spectroscopy. To evaluate photopolymerisation chemical characteristics by the appearance or disappearance of chemical bonds, Fourier Transform Infrared with Attenuated Total Reflectance (FTIR-ATR) spectroscopy (Alpha FT-IR Bruker, Belgium) with a resolution of $4 \mathrm{~cm}^{-1}$ and 64 scans was carried out. Chemical analysis was attempted on the small portion of pre-polymerised solution left in each beaker as well as photopolymerised hydrogels cylinders (Fig. 2). All tests were performed at room temperature (RT), in a spectral range of 400-4000 $\mathrm{cm}^{-1}$. After scanning, relevant picks of absorbance in respect to wavenumber $\left(\mathrm{cm}^{-1}\right)$ were analysed through Opus software.

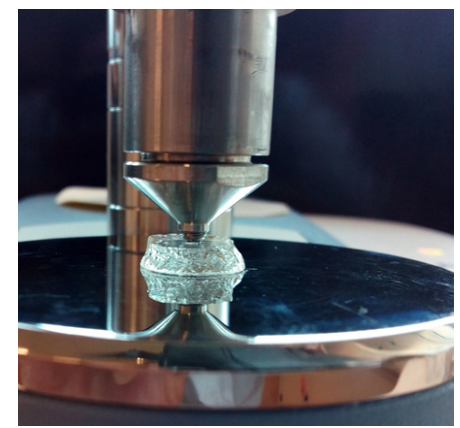

Fig. 2- FTIR procedure for a hydrogel cylinder.

- Swelling studies. Swelling studies consisted on submerging membrane cylinders in water and through successive measurements of their mass, estimate their capacity to retain water, until their stabilization. This way, samples from $A_{1}$ to $C_{3}$ were placed in distilled water to determine their weight (W) of full-wet PEGDMA hydrogel at a given time, after measuring its initial weight (Wi). All tests were performed in triplicate. Water retention was then calculated using Eq. 1.

Water retention $=W / W i$

- Compression resistance tests. Using a mechanical test machine (Instron 4505) compression resistance tests were made (Fig. 3). Compression tests were conducted with a cross-head displacement speed of $1 \mathrm{~mm} / \mathrm{min}$. Each cylinder height was measured before being placed in a support, and vertical down force was loaded by the cell until complete break. Through adequate 
software, successive displacement and force values were saved. For evaluation, Strain (e) (Eq. 2), and Stress $(\sigma)$ (Eq. 3), were calculated and compressive modulus (E) was determined through the Hook's Law of the linear region of the Stress in respect to Strain graphics.

$\varepsilon(\%)=(\delta-\delta i) / h$

where $\delta$ is the stretch at a time, $\delta_{i}$ is the initial stretch, and $h$ is the height of the sample.

$\sigma=F / A$

where $F$ is the force applied on cylinders and $A$ is the area of the previous cylinder mould. Tests were carried on 5 cylinders for each sample and compressive modulus and maximum stress were calculated.

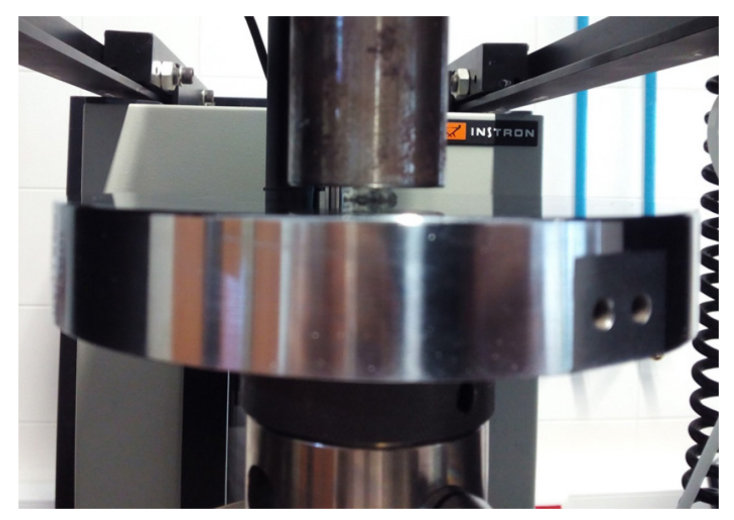

Fig. 3- Mechanical Compression test. Force and displacement data were assessed for subsequent Maximum Stress and Compressive Modulus data.

\section{Results and Discussion}

Characterization of PEGDMA membranes was taken with performance of FTIR-ATR analysis, mechanical compression trials and swelling tests.

FTIR-ATR. To analyse whether increases in photoinitiator or water content led to changes in hydrogel crosslinking, the non-polymerized samples were analysed by Fourier Transform Infrared Spectroscopy and compared to respective UV-cured samples. In figure 4, all A, B and C samples with variations on water content for both non-polymerised (left) and polymerised (right) subfigures present the peak at $1637 \mathrm{~cm}^{-1}$, related with $\mathrm{C}=\mathrm{C}$ bonds. Furthermore, the absorbance or the intensity of the mentioned peak is different. With increase in water content, the intensity of the peak decreases which is related to a fewer quantity of particularly $\mathrm{C}=\mathrm{C}$ bonds by less quantity of PEGDMA. This happens in general for all samples, independently of the concentration of photoinitiator. Besides, with the increase in photoinitiator should exist a trend to decrease the same peak (related with non-polymerisation of PEGDMA), which was only verified on $0 \%$ water samples.

Comparing the same peak on $0 \%$ water samples for non-polymerised results (left subfigure) with polymerised ones (right subfigure) it was found a decrease in $1637 \mathrm{~cm}^{-1}$ peak, independent of photoinitiator content. This could be related to the fact that $\mathrm{A}_{1}, \mathrm{~B}_{1}, \mathrm{C}_{1}$ samples, when polymerised show a decrease in the number of $\mathrm{C}=\mathrm{C}$ peaks, once polymerisation brakes, the bond on acrylate radicals of PEGDMA, according to Lucas and it's colleagues [19]. With free-radical polymerisation, $\mathrm{C}=\mathrm{C}$ tends to decrease, as a consequence of the connection between methacrylate group of PEGDMA and the carbonyl group $\mathrm{C}=\mathrm{O}$ from the photoinitiator Irgacure 2959. In case of samples with $15 \%$ and $30 \%$ water, after the 15 minutes of exposure to UV light, results show an increase of the mentioned peak in photopolymerised graphics. Considering this, it is possible to indicate that 
water prevents polymerisation or crosslinking of functional groups on acrylate radicals of PEGDMA with the free radical carbonyl group from photoinitiator Irgacure 2959.
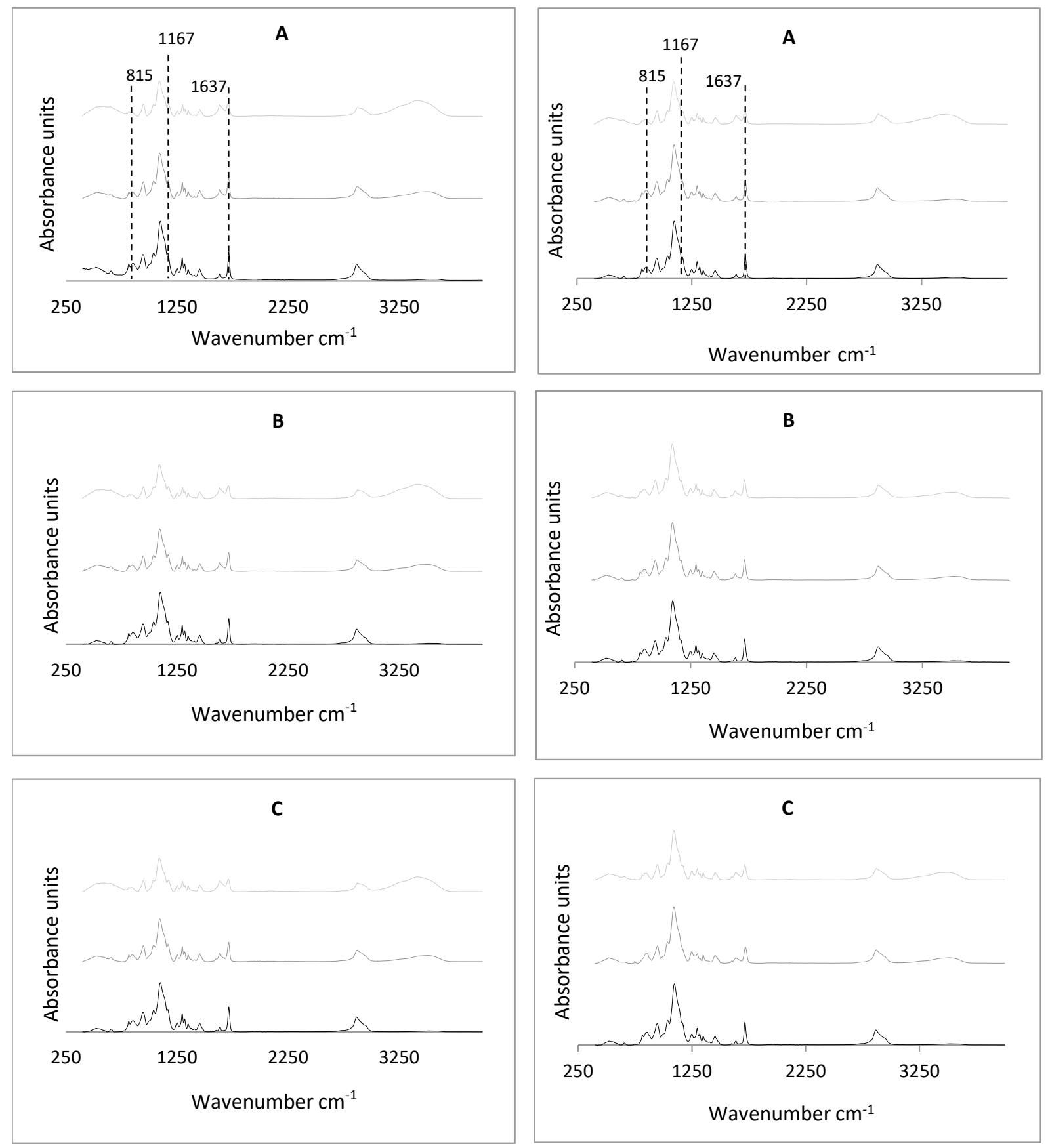

0 (wt\%) water

15 (wt\%) water

30 (wt\%) water

Fig. 4- Absorbance spectrum for non-polymerised samples (left) and polymerised samples (right). In dash are the wavenumbers of peaks related with polymerisation $\left(815,1167\right.$ and $\left.1637 \mathrm{~cm}^{-1}\right)$.

Compression resistance. The typical compression behaviour of PEGDMA hydrogels was achieved by constructing the graphic of Stress in respect to Strain. Figure 5 shows the general compressive behaviour of all hydrogel cylinders from all samples, summarized in table 2 . The behaviour is distinguished in three phases: chain extension corresponding to elastic deformation, chain slipping corresponding to plastic deformation and fracture, which represents the behaviour of an elastomer. A statistical comparison of the tensile testing data $(n=5)$ for each sample was 
performed using a 2-way ANOVA statistical analysis. Differences were considered significant when $p \leq 0.05$. The software package used to perform statistical analysis was Graph Pad, version 6.0.

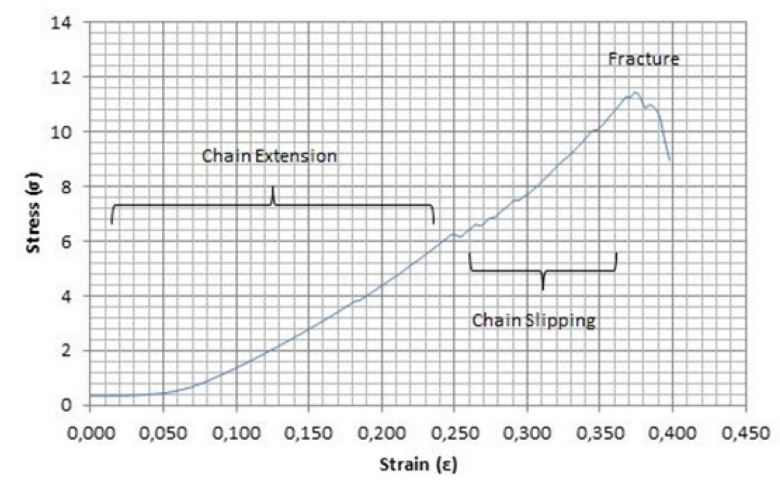

Fig. 5- General obtained compressive behaviour of PEGDMA hydrogels.

The maximum stress and compressive modulus, were then evaluated followed by a 2-way ANOVA statistical analysis according to figure 6 and figure 7 below. The analysis was made evaluating the water and photoinitiator influence separately as two independent variables. Hydrogel sample $\mathrm{A}_{2}$ $(0.5 \%$ photoinitiator, $15 \%$ water $)$ is the one showing the highest maximum stress and the higher compressive modulus of all. On the other side, $\mathrm{A}_{3}(0.5 \%$ photoinitiator, $30 \%$ water $)$ is the one representing the lowest maximum stress and is the less compressive Hydrogel.

Water influence. Relatively to maximum stress (figure 6), and considering now water content variations in A ( $0.5 \%$ photoinitiator) hydrogels, maximum stress rises with increase in water to $15 \%$ $(p<0.001)$ and decreases in 30\% water content $(p<0.001)$. In $\mathrm{B}(1.0 \%$ photoinitiator $)$ no differences between changing the water content were found and all hydrogels show similar behaviour. Relatively to C (1.5\% photoinitiator) hydrogels showed the same behaviour, however, an increase in water content to $15 \%$, reduces significative the maximum stress $(p<0.001)$ and the increase to $30 \%$ slightly increases maximum stress, beyond considering the increase less significant $(p<0.01)$.

In figure 7 , analysing A ( $0.5 \%$ photoinitiator $)$ hydrogels, the increase in water content to $15 \%$ water of total solution concentration increases compressive modulus to a very high value $(p<0.0001)$, but further increase in water content from $15 \%$ to $30 \%$ reduces compressive modulus to lowest value (p $<0.0001)$. In case of hydrogels B $(1.0 \%$ photoinitiator), the same rise of water content to $15 \%$ and after $30 \%$ greatly reduces the compressive modulus $(p<0.0001)$ despite the fall between $15 \%$ and $30 \%$ having no significance. Similarly, in hydrogels $C$ (1.5\% photoinitiator), the increase in water content from $0 \%$ to $15 \%$ and after $30 \%$ decreases the compressive modulus to low values $(p<$ $0.0001)$ although the decrease verified between $15 \%$ and $30 \%$ be less significative $(p<0.05)$.

Photoinitiator influence. The increase in photoinitiator to $1.0 \%$ in $1(0 \%$ water) hydrogels reveals no difference in maximum stress value, although with increase in photoinitiator content to $1.5 \%$, maximum stress increases $(p<0.05)$. However, in $2(15 \%$ water $)$ hydrogels, a general decrease is verified $(p<0.0001)$ while in $3(30 \%$ water) hydrogels there is no difference found in maximum stress once the values are very similar.

Focusing now on compressive modulus (figure 7), for hydrogels with no water (1), the increase in photoinitiator content contributes to rise the compressive modulus despite the weak raise $(p<0.05)$. For $2(15 \%$ water) hydrogels, increasing photoinitiator content tends to decrease a lot the compressive modulus $(p<0.0001)$ not being verified differences between $1.0 \%$ and $1.5 \%$ hydrogels. Finally, for 3 (30\% water) hydrogels, the increase in photoinitiator content to $1.0 \%$ increases the compressive modulus $(p<0.01)$ while to $1.5 \%$ no differences were found. 


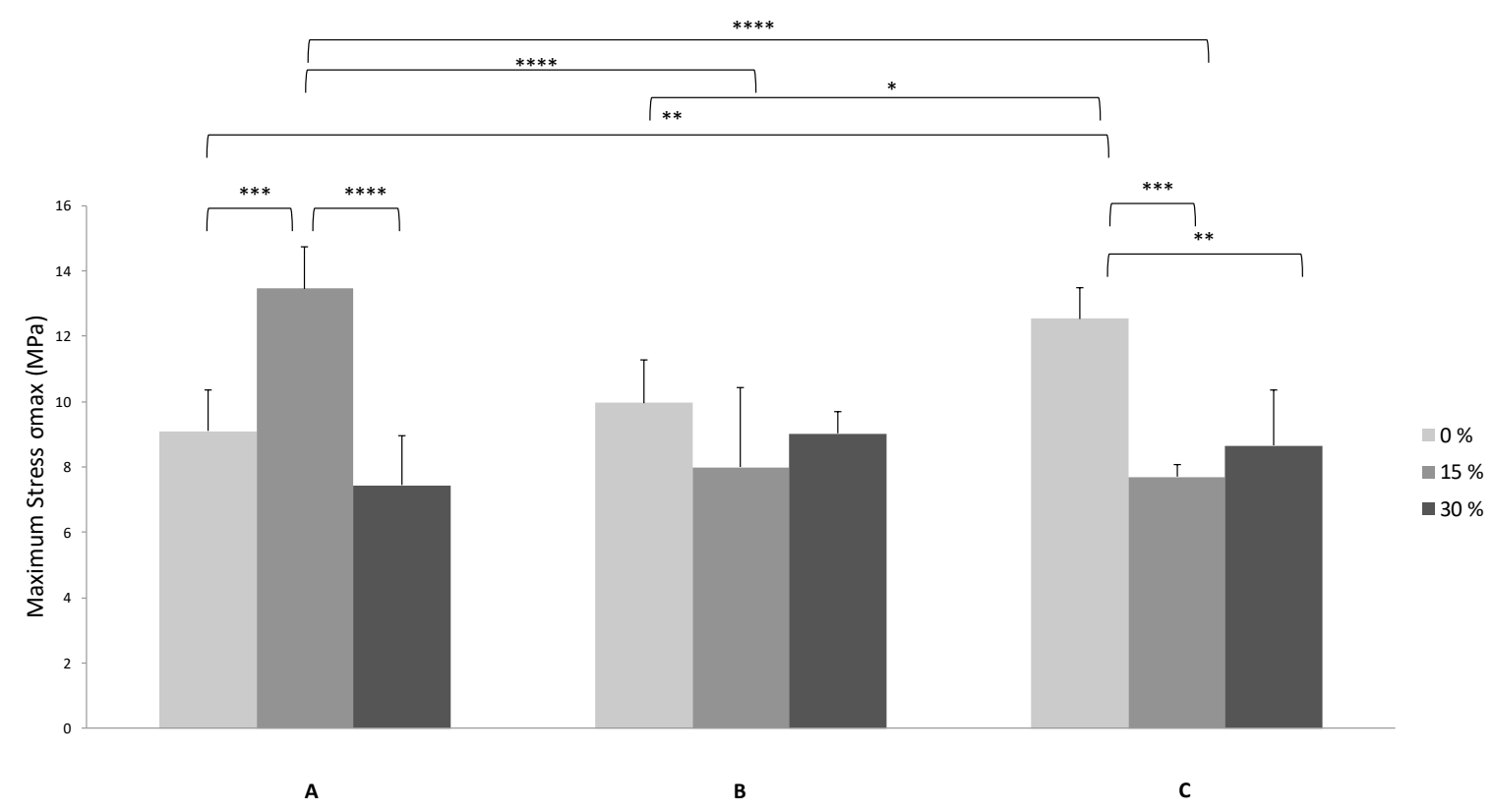

Fig. 6- Maximum Stress for PEGDMA 750 samples, considering the photoinitiator content (wt\%), $0.5,1$ and 1.5 represented respectively by $\mathrm{A}, \mathrm{B}$ and $\mathrm{C}$; and water content 0,15 , and 30 (wt\%), by increased grey scales.

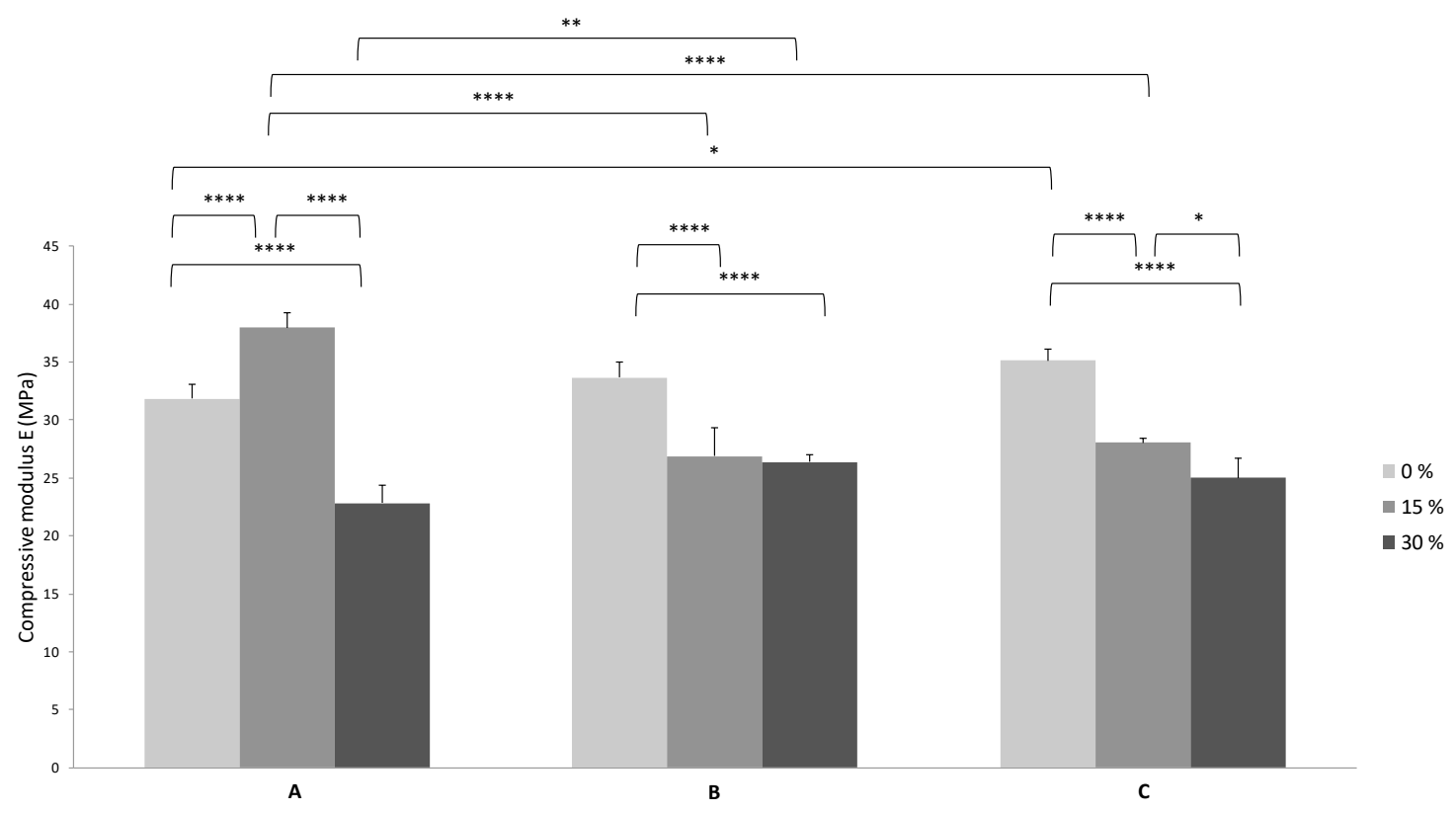

Fig. 7- Compressive modulus values for PEGDMA 750 hydrogels, considering the photoinitiator content (wt $\%$ ), 0.5, 1 and 1.5 represented respectively by $\mathrm{A}, \mathrm{B}$ and $\mathrm{C}$; and water content 0,15 , and $30(\mathrm{wt} \%)$, by increased grey scales. 
Table 2-Mechanical tests of each PEGDMA 750 hydrogel. Maximum stress is represented by $\sigma \max$ $(\mathrm{MPa})$, Strain $\varepsilon(\%)$, and Compressive modulus by $\mathrm{E}(\mathrm{MPa})$.

\section{Water content $(\mathrm{Wt} \%)$}

\begin{tabular}{|c|c|c|c|c|c|c|c|c|c|}
\hline & \multicolumn{3}{|c|}{$\mathbf{0}$} & \multicolumn{3}{|c|}{15} & \multicolumn{3}{|c|}{30} \\
\hline $\begin{array}{l}\text { Irgacure } \\
2959 \\
(\mathbf{W t} \%)\end{array}$ & $\sigma_{\max }(\mathrm{MPa})$ & $\varepsilon(\%)$ & $\mathrm{E}(\mathrm{MPa})$ & $\begin{array}{c}\sigma_{\max } \\
(\mathrm{MPa})\end{array}$ & $\varepsilon(\%)$ & $\mathrm{E}(\mathrm{MPa})$ & $\begin{array}{c}\sigma_{\max } \\
(\mathrm{MPa})\end{array}$ & $\varepsilon(\%)$ & $\mathrm{E}(\mathrm{MPa})$ \\
\hline 0,5 & $\begin{array}{c}9,09 \pm \\
1,27\end{array}$ & $\begin{array}{c}28,67 \pm \\
0,01\end{array}$ & $\begin{array}{c}31,84 \pm \\
1,34\end{array}$ & $\begin{array}{c}13,45 \pm \\
1,30\end{array}$ & $\begin{array}{c}33,30 \pm \\
0,02\end{array}$ & $\begin{array}{c}37,96 \pm \\
2,45\end{array}$ & $\begin{array}{c}7,44 \pm \pm \\
1,52\end{array}$ & $\begin{array}{c}28,93 \pm \\
0,02\end{array}$ & $\begin{array}{c}22,84 \pm \\
0,69\end{array}$ \\
\hline 1 & $\begin{array}{r}9,95 \pm \\
0,96\end{array}$ & $\begin{array}{c}29,63 \pm \\
0,04\end{array}$ & $\begin{array}{c}33,65 \pm \\
1,81\end{array}$ & $\begin{array}{c}7,99 \pm \\
0,39\end{array}$ & $\begin{array}{c}32,47 \pm \\
0,003\end{array}$ & $\begin{array}{c}26,9 \pm \\
0,67\end{array}$ & $\begin{array}{c}9,02 \pm \\
1,68\end{array}$ & $\begin{array}{c}31,90 \pm \\
0,02\end{array}$ & $\begin{array}{c}26,37 \pm \\
0,18\end{array}$ \\
\hline 1,5 & $\begin{array}{c}12,53 \pm \\
1,25\end{array}$ & $\begin{array}{c}33,80 \pm \\
0,01\end{array}$ & $\begin{array}{c}35,11 \pm \\
0,87\end{array}$ & $\begin{array}{l}7,7 \pm \\
1,01\end{array}$ & $\begin{array}{c}30,07 \pm \\
0,017\end{array}$ & $\begin{array}{c}28,05 \pm \\
0,73\end{array}$ & $\begin{array}{c}8,66 \pm \pm \\
0,97\end{array}$ & $\begin{array}{c}31,33 \pm \\
0,03\end{array}$ & $\begin{array}{c}25,00 \pm \\
0,98\end{array}$ \\
\hline
\end{tabular}

Swelling. Almost all hydrogels tend to stabilize at 1.4 to 1.6 times their initial weight. That means they can absorb water approximately $40-60 \%$ their initial weight.

As we can see in figure 8 , at a given time nearly 0 almost all hydrogels absorb $20 \%$ of their weight in water, and keep absorbing it until they reach the maximum water retention. Then, the water retention decreases to stabilize the osmotic pressure and the hydrogel enters in stabilization.
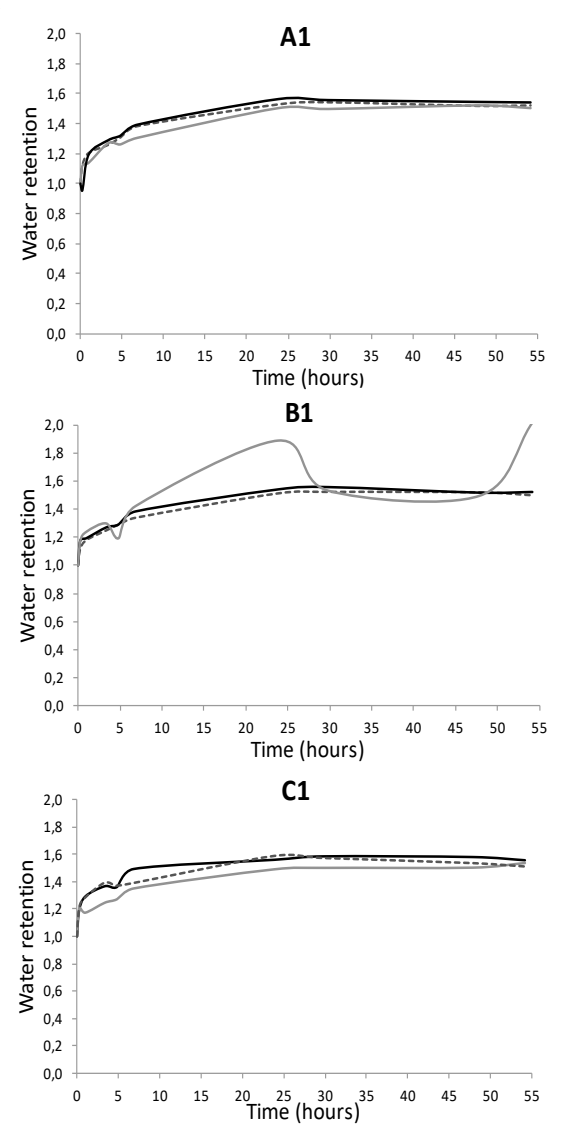

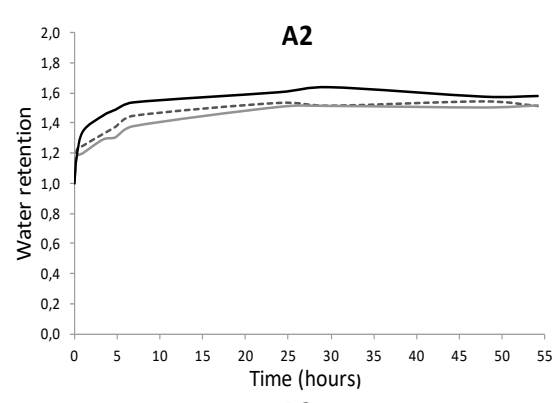

B2

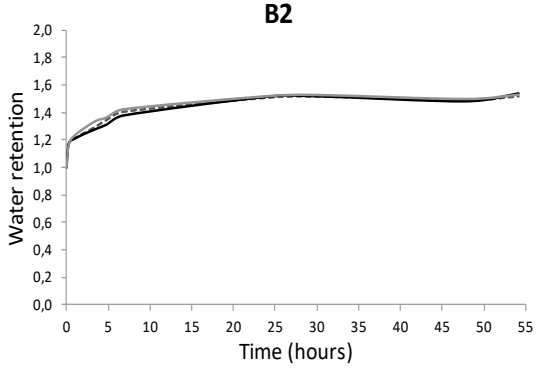

C2

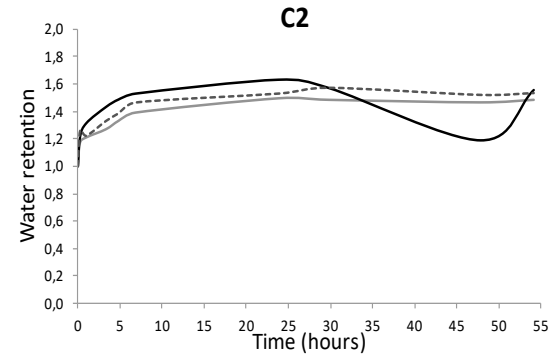

A3

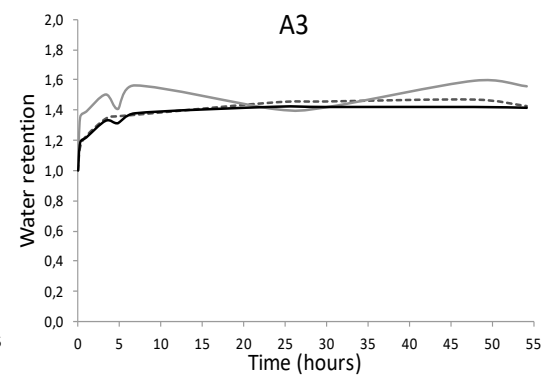

B3

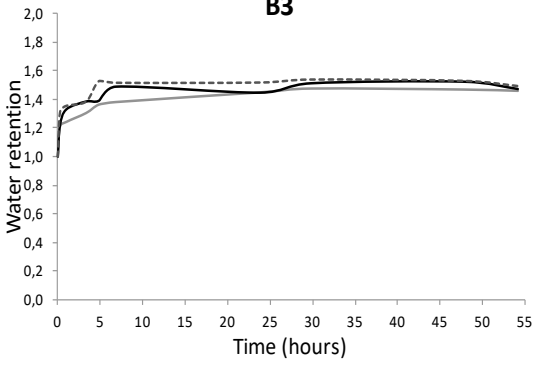

C3

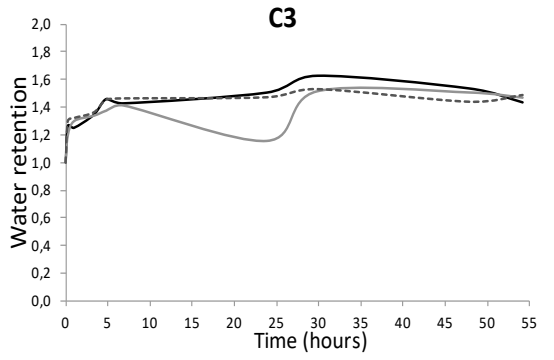

Fig. 8- Water retention as function of time for all hydrogels. Each hydrogel was assessed by testing three cylinders represented by the different dashes. Horizontal alignment: A, B, C (photoinitiator content (wt\%): 0.5, 1.0, 1.5 respectively). Vertical alignment: 1, 2, 3 (water content (wt\%): 0; 15; 30 respectively. 
This time, statistical analysis $(\mathrm{n}=3)$ for each sample were performed using a 1-way ANOVA, with the water retention variation as the only variable to evaluate. According to figure 8 , all hydrogels have approximately the same water retention $\sim 50 \%-60 \%$ of their initial weight. Beyond that, some differences still persist between $\mathrm{A}_{3}$ hydrogel and all the other hydrogels $(p<0.05)$ particularly with $\mathrm{C}_{1}(p<0.01)$ and $\mathrm{C}_{3}(p<0.001)$ as well as between $\mathrm{C}_{2}$ and $\mathrm{C}_{3}$ hydrogels $(p<0.05)$. This means that only $\mathrm{A}_{3}$ has a different water retention capacity and also that, in $\mathrm{C}(1.5 \%$ photoinitiator $)$ hydrogels the increase of water from $15 \%$ to $30 \%$ increases the water retention.

Hydrogels $\mathrm{A}_{3}$ and $\mathrm{B}_{3}$ are the ones with less capability for water retention with values of retention nearly $40 \%$ and $50 \%$ respectively of their initial weight. This fact could be related to their high amount of water (30\% water) in comparison to the other hydrogels. But, $\mathrm{C}_{3}$ is the one showing the higher value of water retention therefore indicating that polymerization induces or increases the capability of hydrogels to retain water.

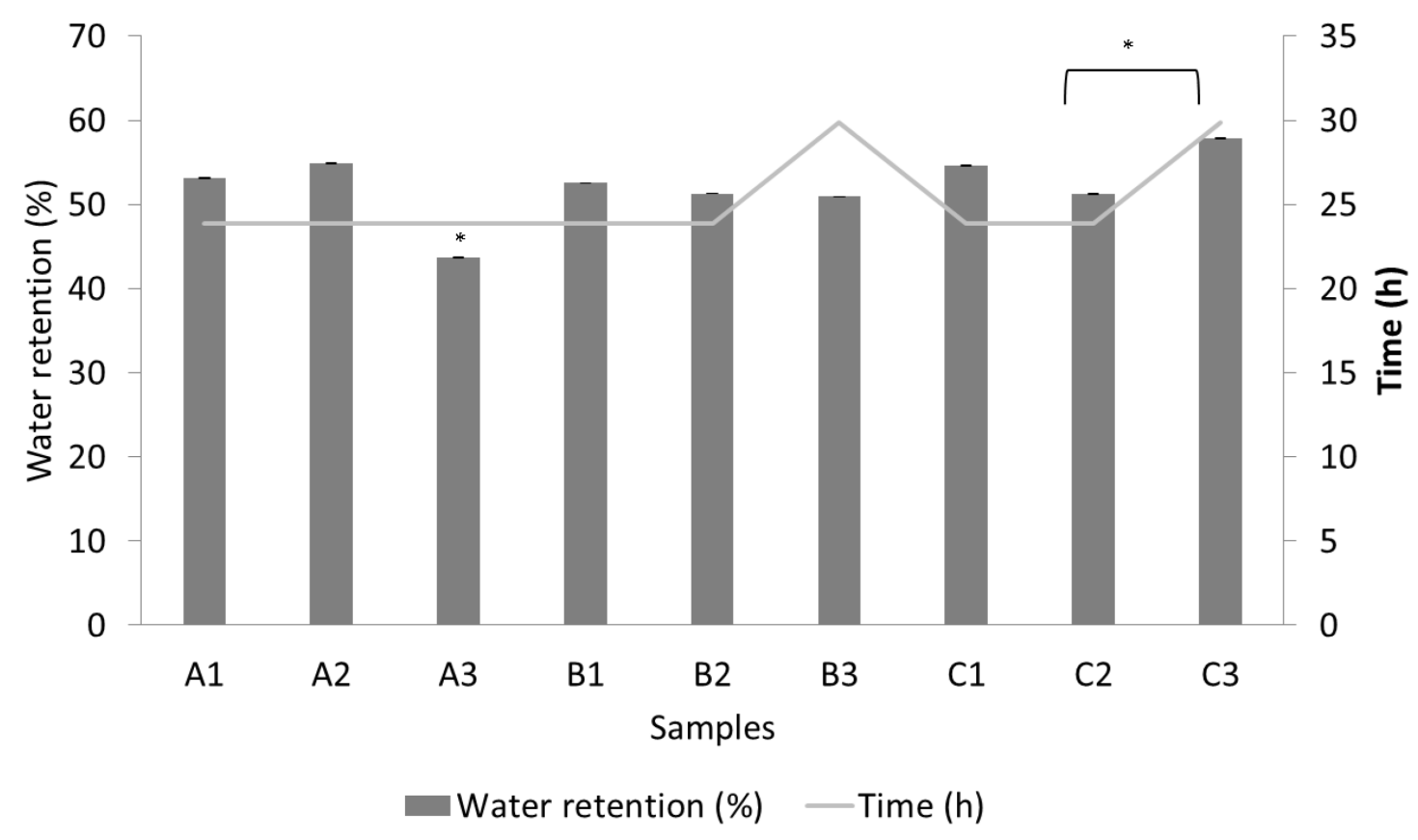

Fig. 9- Water retention capacity for each PEGDMA hydrogels and time stabilization. ANOVA results show relations between hydrogels.

\section{Summary}

The increase in photoinitiator induces polymerisation on $0 \%$ water samples, verified by the decrease in the peak $1637 \mathrm{~cm}^{-1}$ by disappearance of $\mathrm{C}=\mathrm{C}$ bonds at FTIR-ATR analysis. The increase in water content prevents polymerisation. Furthermore, the incorporation of photoinitiator at hydrogels with no water, increase the maximum stress, because hydrogels have a more polymerised structure, leading to a more cross-linked network that can sustain increase charges.

Water incorporation in hydrogels causes a decrease at maximum stress, since water prevents polymerisation, leading to a lack of cross-linked network that can sustain better the applied charges, although hydrogels with more photoinitiator content show a more polymerised network than the others and retain more water in the existent network. In case of hydrogel A2 $(0.5 \%$ photoinitiator, $15 \%$ water) as it belongs to the less polymerised hydrogels (A), the incorporation of $15 \%$ of water could be the cause for it not retaining water in its structure sustaining the pressure only with high compressive water and essentially hydrogel solution (not polymerized) what may suggest reducing the tenacity of the hydrogel and increasing its fragility more than A3 hydrogel even that the superior amount of water had prevented a well polymerisation. The slightly rise of water from $15 \%$ to $30 \%$ 
with increase in photoinitiator content to $1,0 \%$ and also $1,5 \%$ does not change much the maximum stress values.

In respect to compression data, it is possible to conclude that increasing the amount of photoinitiator for no water content hydrogels increases their compressive modulus what may suggest that more polymerised samples or higher cross-linked networks are stronger and sustain more higher stress being less strained (more resistant) without water in their chains. Similarly to maximum stress, the incorporation of water does that compressive modulus decreases with the exception of $\mathrm{A}_{2}$ hydrogel for the reasons mentioned before which might suggest a correlation between maximum stress and compressive modulus. The difference resides in increasing the water content to $30 \%$ which keeps the maximum stress more or less the same when compared to $15 \%$ water hydrogels but showing a less compressive behaviour than $15 \%$ water hydrogels indicating that they can sustain the same charges but break more easily, which is related to an increase in fragility or a decrease in tenacity. Comparing all strains, we can identify that all samples tend to break at $30 \%$ deformation of their initial length. That is, all samples can hold the stress being applied until the hydrogel reaches $2 / 3$ of its initial height.

Finally, water retention tells us that in fact hydrogels can be hydrated few times their weight but showing no significative differences between them on the capacity to retain water. Beyond that, hydrogels with more water content tend to take more time to stabilize.

\section{Acknowledgements}

This work is supported by the Fundação para a Ciência e a Tecnologia (FCT) and Centro2020 through the Project references: UID/Multi/04044/2013 and PAMI - ROTEIRO/0328/2013 ( $\mathrm{N}^{\circ}$ 022158). It is also funded by the projects insitu.Biomas (POCI-01-0247-FEDER-017771), and NEXT. parts (POCI-01-0247-FEDER-017963) from the Portuguese National Innovation Agency.

\section{References}

[1] S.J. Lee, J.J. Yoo, A. Atala, Biomaterials and Tissue Engineering, in: B.W. Kim (Eds.), Clinical Regenerative Medicine in Urology, Springer Singapore, Singapore, 2018, pp. 17-51.

[2] M. Lutolf, J. Hubbell, Synthetic biomaterials as instructive extracellular microenvironments for morphogenesis in tissue engineering, Nature biotechnology 23 (2005) 47.

[3] D.F. Williams, The Biomaterials Conundrum in Tissue Engineering, Tissue Engineering Part A 20 (2014) 1129-1131.

[4] D. Williams, Essential Biomaterials Science, ed., 2014.

[5] I.M. El-Sherbiny, M.H. Yacoub, Hydrogel scaffolds for tissue engineering: Progress and challenges, Global Cardiology Science and Practice (2013) 38.

[6] A. Gutowska, B. Jeong, M. Jasionowski, Injectable gels for tissue engineering, The Anatomical Record 263 (2001) 342-349.

[7] M.C. Cushing, K.S. Anseth, Hydrogel cell cultures, Science 316 (2007) 1133-1134.

[8] J.A. Killion, L.M. Geever, D.M. Devine, J.E. Kennedy , C.L. Higginbotham, Mechanical properties and thermal behaviour of PEGDMA hydrogels for potential bone regeneration application, Journal of the mechanical behavior of biomedical materials 4 (2011) 1219-1227.

[9] S. Khalil, W. Sun, Bioprinting endothelial cells with alginate for 3D tissue constructs, Journal of biomechanical engineering 131 (2009) 111002.

[10] J. Malda, J. Visser, F.P. Melchels, T. Jüngst, W.E. Hennink, W.J. Dhert, J. Groll , D.W. Hutmacher, 25th anniversary article: engineering hydrogels for biofabrication, Advanced materials 25 (2013) 5011-5028. 
[11] J.J. Rice, M.M. Martino, L. De Laporte, F. Tortelli, P.S. Briquez, J.A. Hubbell, Engineering the regenerative microenvironment with biomaterials, Advanced healthcare materials 2 (2013) 5771.

[12] K.B. Fonseca, P.L. Granja , C.C. Barrias, Engineering proteolytically-degradable artificial extracellular matrices, Progress in Polymer Science 39 (2014) 2010-2029.

[13] A. Skardal , A. Atala, Biomaterials for integration with 3-D bioprinting, Annals of biomedical engineering 43 (2015) 730-746.

[14] K.T. Nguyen , J.L. West, Photopolymerizable hydrogels for tissue engineering applications, Biomaterials 23 (2002) 4307-4314.

[15] I. Mironi-Harpaz, D.Y. Wang, S. Venkatraman , D. Seliktar, Photopolymerization of cellencapsulating hydrogels: crosslinking efficiency versus cytotoxicity, Acta biomaterialia 8 (2012) 1838-1848.

[16] R.F. Pereira , P.J. Bártolo, 3D bioprinting of photocrosslinkable hydrogel constructs, Journal of Applied Polymer Science 132 (2015)

[17] L.F. Zhang, R. Sun, L. Xu, J. Du, Z.C. Xiong, H.C. Chen , C.D. Xiong, Hydrophilic poly (ethylene glycol) coating on PDLLA/BCP bone scaffold for drug delivery and cell culture, Materials Science and Engineering: C 28 (2008) 141-149.

[18] G. Ma, D. Yang, Q. Li, K. Wang, B. Chen, J.F. Kennedy, J. Nie, Injectable hydrogels based on chitosan derivative/polyethylene glycol dimethacrylate/N, N-dimethylacrylamide as bone tissue engineering matrix, Carbohydrate Polymers 79 (2010) 620-627.

[19] V. Lucas, Controlled migration of cells on mechanically, physically and chemically patterned biomaterials, (2015) 